(C) 2010 IEEE. Personal use of this material is permitted. Permission from IEEE must be obtained for all other uses, in any current or future media, including reprinting/republishing this material for advertising or promotional purposes, creating new collective works, for resale or redistribution to servers or lists, or reuse of any copyrighted component of this work in other works. 


\title{
Effects of the Super Bialkali Photocathode on the Performance Characteristics of a Position-Sensitive Depth-of-Interaction PET Detector Module
}

\author{
Juan J. Vaquero, Senior Member, IEEE, José M. Udías, Member, IEEE, Jurgen Seidel, Member, IEEE, \\ Samuel España, and Manuel Desco
}

\begin{abstract}
Super Bialkali (SBA) photocathode is a new technology that improves the spectral response characteristics of position sensitive PMTs, boosting their quantum efficiency up to $35 \%$. In this experiment, two SBA tubes were introduced into a production line of PET detectors mixed with the regular tubes. The detectors were assembled using the standard factory protocols for detector mounting, calibration and testing. We report an evaluation of the improvement introduced by the SBA photocathode comparing the spatial and energy resolution and the depth-of-interaction (DOI) performance of PET detector modules with DOI capabilities. We conclude that the superior performance of the SBA tube may enable the use of arrays with a larger number of crystals of smaller footprint, thus potentially improving the detector intrinsic spatial resolution without degrading the energy resolution or the phoswich (DOI) discrimination capability.
\end{abstract}

Index Terms-Gamma-ray detector, PET, photomultiplier.

\section{INTRODUCTION}

$\mathbf{P}$ HOTOMULTIPLIERS (PMT) convert the incident light photons produced by the crystal scintillator into electrons by means of a photoemission process that occurs in the photocathode. The efficiency of this process depends on several

Manuscript received October 13, 2009; revised January 26, 2010; accepted May 26, 2010. Date of publication July 19, 2010; date of current version October 15, 2010. This work was supported in part by the CENIT Program (Ministerio de Industria), Comunidad de Madrid program ARTEMIS-P2009/DPI-1802, TEC2007-64731/TCM (Ministerio de Educación y Ciencia), and the RETIC-RECAVA, ISCIII. The contribution of J. Seidel wa supported in whole or in part with federal funds from the National Cancer Institute, National Institutes of Health, under Contract HHSN261200800001E. The content of this work does not necessarily reflect the views or policies of the Department of Health and Human Services, nor does mention of trade names, commercial products, or organizations imply endorsement by the U.S. Government.

J. J. Vaquero is with the Unidad de Medicina y Cirugía Experimental, Hospital General Universitario Gregorio Marañón, E-28007 Madrid, Spain (e-mail: juanjo@mce.hggm.es).

J. M. Udías and S. España are with the Departamento de Física Atómica, Grupo de Física Nuclear, Molecular y Nuclear, Universidad Complutense, E-28040 Madrid, Spain (e-mail: jose@nuc2.fis.ucm.es, samuel@nuc1.fis.ucm.es).

J. Seidel is with the Molecular Imaging Program, National Cancer Institute, National Institutes of Health, Bethesda, MD 20892 USA - per contract with SAIC-Frederick Inc., Frederick, MD 21702 USA (e-mail: johnnybgoode@mac. com).

M. Desco is with the Unidad de Medicina y Cirugía Experimental, Hospital General Universitario Gregorio Marañón, E-28007 Madrid, Spain and also with the Departamento de Bioingeniería, Universidad Carlos III, E-28911 Madrid, Spain (e-mail: desco@mce.hggm.es). physical phenomena well described in [1]. In terms of scintillation counting the most relevant parameters are the spectral response and the quantum efficiency (QE), the latter defined as the number of photoelectrons emitted divided by the number of incident photons. This energy conversion is followed by an electron multiplication that results in a charge pulse at the final anode. Since the photoelectron conversion represents the first step in the amplification chain, any improvement in the QE should benefit the resulting overall performance of the PMT.

Super Bialkali (SBA) and Ultra Bialkali (UBA) photocathodes are new technologies that improve the spectral response characteristics of position sensitive PMTs, boosting their quantum efficiency up to $35 \%$ and $43 \%$ (typ.), respectively, compared to regular bialkali (BA) photocathodes with a QE of 25\% (typ.) [2].

In this work we report an evaluation of this improvement, of which preliminary results were presented in [3], by comparing the spatial and energy resolution and the depth-of-interaction performance of PET detector modules assembled using the regular and the SBA versions of comparable PS-PMTs.

\section{Materials AND MethodS}

\section{A. Detector Modules}

Two SBA tubes (Hamamatsu R8900-100-C12) were introduced into a production line of PET detectors (SEDECAL, Madrid, Spain) mixed with the regular tubes (Hamamatsu R8520-00-C12). The detectors were assembled on the same day and by the same operator using the standard factory protocols for detector mounting, calibration and testing. The module comprises two more parts: the above mentioned PS-PMT is coupled to a phoswich scintillator crystal array (LYSO , and GSO

$1.45 \mathrm{~mm} \times 1.45$ mim which meach phoswich

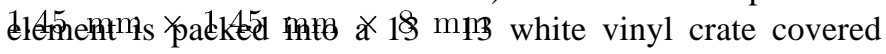
at the entrance end with PTFE tape to enhance reflection of scintillation light onto the PS-PMT photocathode [4]. The dual-scintillator phoswich was created by optically joining together end-to-end two different scintillators (all faces chemically etched): cerium-doped lutetium-yttrium orthosilicate (LYSO, Lu Y SiO :Ce) in the front crystal layer, and cerium-dopady godolinimm orthosilicate (GSO, Gd SiO :Ce) in the rear layer. The two crystals glued into single2phoswich elements can be distinguished by the detector electronics by measuring their different characteristic light decay time (LYSO, 40 ns; GSO, 60 ns) [5]. 


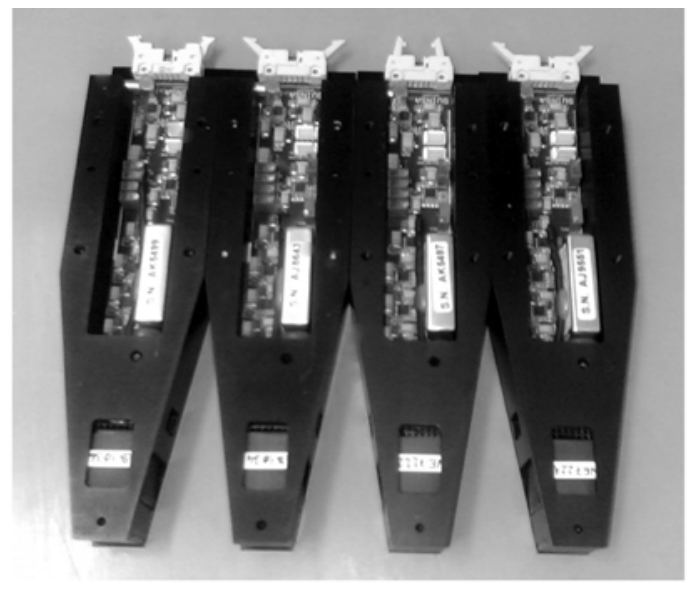

SPECTRAL RESPONSE CHARACTERISTICS Metal Package PMT (TO-8 Type)

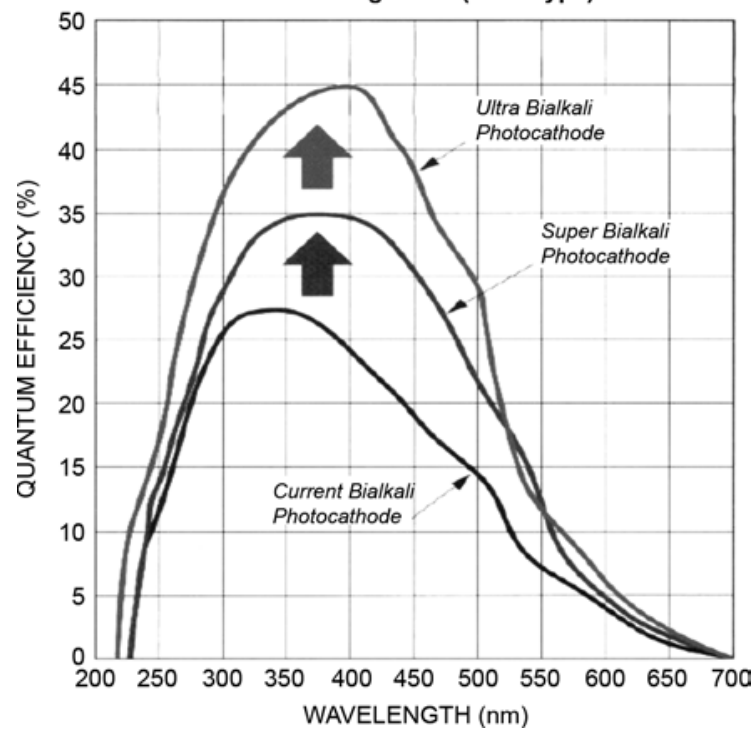

Fig. 1. Finished detector modules as they come out of the production line (upper panel). QE curves for the regular, SBA and UBA PS-PMTs from Hamamatsu (lower panel).

Finally, a single printed circuit board integrates all the electronic circuits for generating the high voltage (HV), the pre-amplification of the position signals, a timing signal derived from the sum of the four position signals, and an additional total energy signal used for the phoswich discrimination. This circuit is attached to the back of the PMTs.

\section{B. Data Acquisition and Processing}

Data acquisition was performed using the same equipment and settings for all the detectors: the 12 anode outputs (six for $\mathrm{X}$ and six for $\mathrm{Y}$ ) were fed to a resistive divider that implements an Anger-like processing [6]-[8]; the resulting four signals that locate the event within the field-of-view (FOV) are digitized using a charge-integration ADC. The event position is calculated with a resolution of eight bits, resulting on a $256 \times 256$ pixel detector image. A delayed sum (energy) signal is also generated by summing the position signals, and is digitized at the same time. LYSO and GSO events are discriminated from one another by applying the "delayed charge integration" technique, an algorithm based on the different light decay constants between the two scintillators to identify the layer of interaction (Depth Of Interaction, DOI) [9]. The DOI of the event is obtained by manipulating the two energy signals (the original and the delayed one) in such a way that whereas the first one is fully integrated, the delayed signal is only partially integrated at its tail section. Since the residual energy signal in that tail section depends on the characteristic light decay time of the crystal (LYSO, $40 \mathrm{~ns}$; GSO, $60 \mathrm{~ns}$ ), the ratio between the delayed integral and the full integral identifies the crystal of interaction, and therefore determines the DOI [10].

The data acquisition protocol was identical for all the detectors: the lower threshold electronic cut-off was the same for all the tubes, and there was no upper-level cut-off. Tube equalization was achieved by modulating each individual $\mathrm{HV}$ forcing the output signals to cover at least two thirds of the digital-to-analog converter dynamic range.

The images from the four detectors were analyzed to measure intrinsic module spatial resolution, energy resolution and DOI performance by using field flood images of a flat ${ }^{68} \mathrm{Ge}$ source.

Intrinsic module resolution was quantified by measuring peak-to-valley ratios and peak separation on different profiles across the PMTs field flood images [11], and measuring the "packing" coefficient. This is calculated from a profile across the central row of a field-flood image, fitting a Gaussian to each individual crystal. The packing coefficient is computed dividing the mean FWHM of the individual crystals by the average separation between them, measured after the fitting as the distance between the centers of the Gaussian peaks of adjacent crystals. In case of Gaussian peaks and a packing coefficient of 1.0 , for example, the peaks are overlapping to a large degree and can barely be distinguished as separate from each other. On the other hand, a packing coefficient of 0.5 or smaller means that the crystals Gaussian profiles are overlapping by $2 \%$ or less. Packing coefficients of 0.5 or smaller are highly desirable to minimize crystal misidentification.

Energy resolution was measured for each crystal separately by fitting a Gaussian function to the photopeak, after discounting the scatter contribution. A boxed factorial ANOVA analysis was applied to all the crystal energy resolutions (1352 in total: $13 \times 13$ phoswich elements, two crystals per phoswich element, four tubes), and the results were represented in a box-and-whisker plot. SBA tubes were tracked by their serial number and identified only at the end of the experiment.

\section{RESULTS}

SBA PMT images in Fig. 2 (left) clearly show the "connectthe-dot" patterns described in [11], whereas they are more difficult to appreciate in the images from the regular PMTs (right). Fig. 3 depicts two central intensity profiles.

Peak-to-valley ratio and peak spacing are quantified in Table I and show that the SD of the peak spacing for both SBA tubes are smaller. All rows have been included for the statistic analysis. The packing coefficient for the SBA tube is 0.197 compared with 0.271 for the regular one, which represents a $27 \%$ improvement.

Crystal maps resulting from the segmentation of the ${ }^{68} \mathrm{Ge}$ field flood images are shown in Fig. 4 (upper panel). Another parameter derived from these field flood images is the inter-crystal 


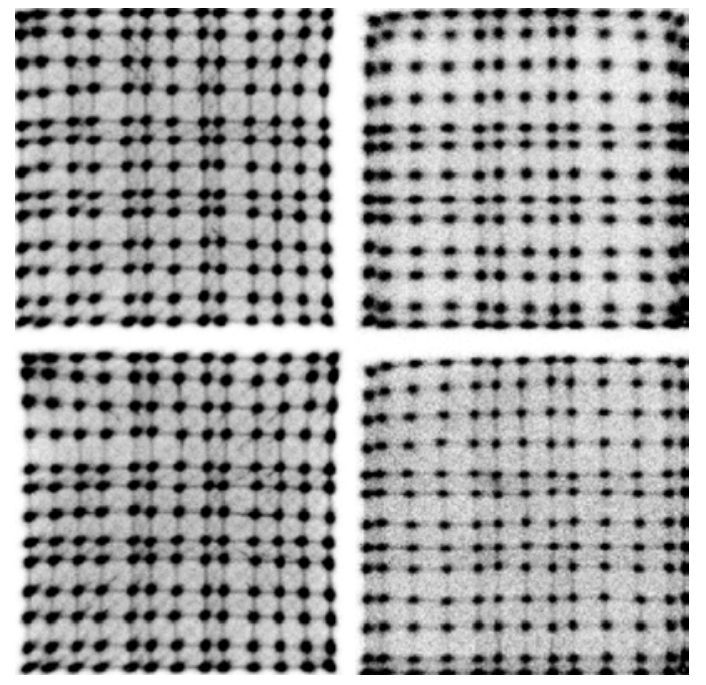

Fig. 2. Field flood images for a ${ }^{68} \mathrm{Ge}$ source illumination. All images were acquired with the same settings. Left column are the SBA tubes, right column the regular ones.

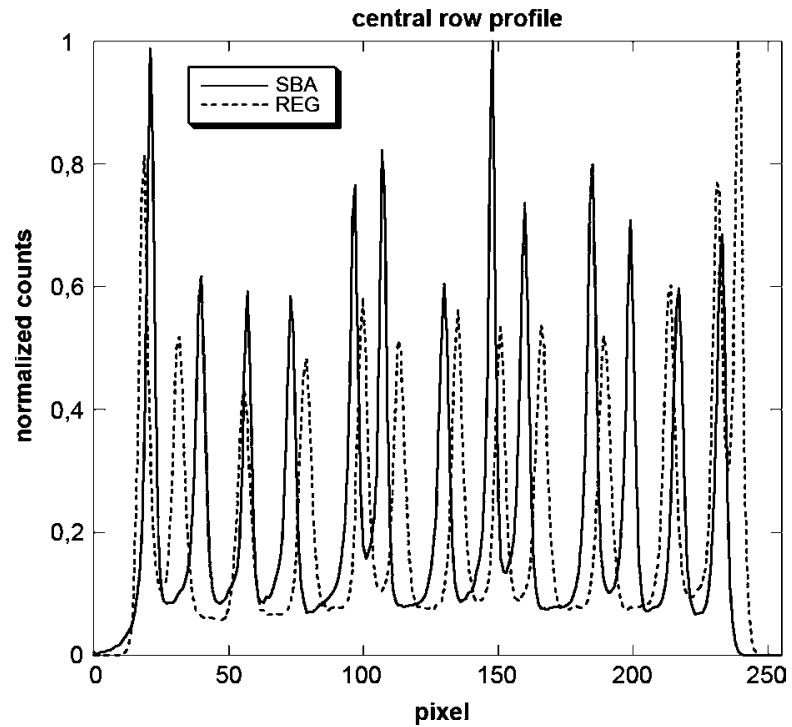

Fig. 3. Central row profile for a field flood illumination with ${ }^{68} \mathrm{Ge}$.

TABLE I

Peak Spacing at the Central Profile of the ${ }^{68}$ Ge Field Flood Image (Median $\pm s d$, in Pixels), Peak-To-Valley Ratio From the Same Profile (Median \pm sd), and Packing CoefFicient (Mean).

\begin{tabular}{ccccc} 
tube & SBA & SBA & Reg. & Reg. \\
\hline peak spacing & $16.7 \pm 4.6$ & $17.8 \pm 3.0$ & $20.1 \pm 5.6$ & $19.8 \pm 5.3$ \\
peak/valley & $8.6 \pm 2.1$ & $7.8 \pm 2.1$ & $6.9 \pm 1.6$ & $7.0 \pm 2.1$ \\
packing coeff. & \multicolumn{2}{c}{0.197} & \multicolumn{2}{c}{0.271}
\end{tabular}

distance variation. Since all the crystals of the scintillator array are equally spaced, it is desired to have a minimum variation on the image points that represent the crystal centers. The lower panel in Fig. 4 shows that while the average peak spacing is similar for both kind of tubes, its dispersion is lower for the SBA; this is more noticeable at the edges of the tube.

The statistical results of energy resolutions measured for each crystal of each detector assembly are shown in Fig. 5. For each
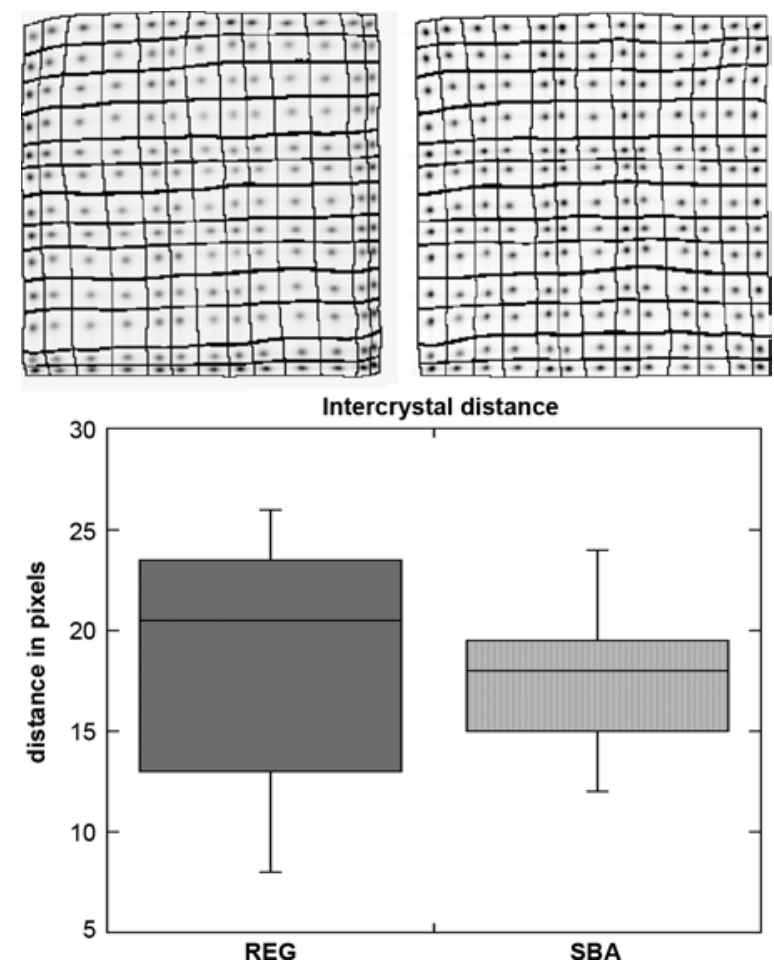

Fig. 4. Crystal maps obtained from the ${ }^{68} \mathrm{Ge}$ field flood images; regular tube on the left, SBA tube on the right, (upper panel). Box plot for the average intercrystal distances for the three central rows of each tube.

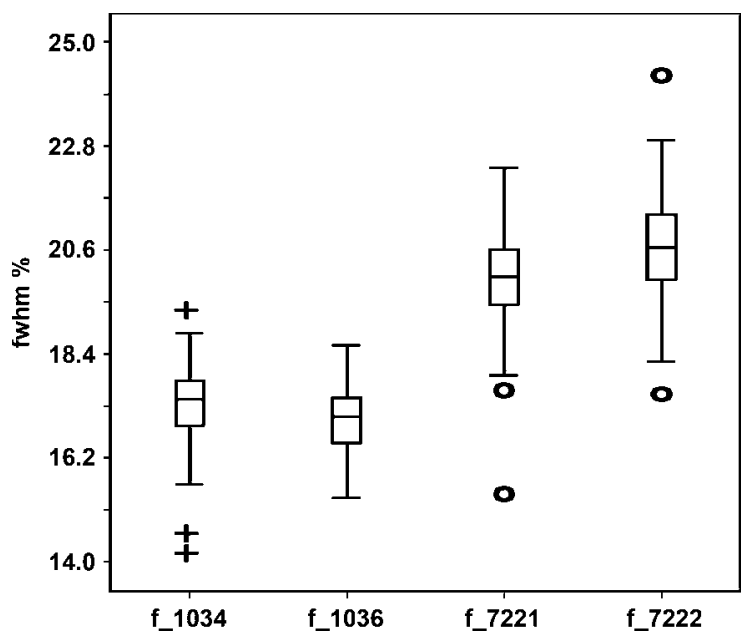

Fig. 5. Box and whiskers plot for the individual LYSO crystals energy resolution, for a ${ }^{68} \mathrm{Ge}$ field flood illumination (SBA: $\mathrm{f} \_1034, \mathrm{f} \_1036$, reg.: $\mathrm{f} \_7221$, f_7222). Statistical difference was significant $(p<0.0001)$. Crosses and open circles represent outliers.

PMT, boxes representing the two intermediate quartiles of the FWHM in percentage are plotted. The whiskers represent the remaining two outer quartiles, whereas the "+" and "o" symbols represent outliers.

Fig. 6 depicts the result of the "delayed charge integration" method used to discriminate between the phoswich crystals. The plot is a histogram of the ratio between the delayed charge and the full charge. The two peaks on the plot represent in the horizontal axis the footprint of the ratios between the total and delayed energy signals, whereas the vertical axis shows the 


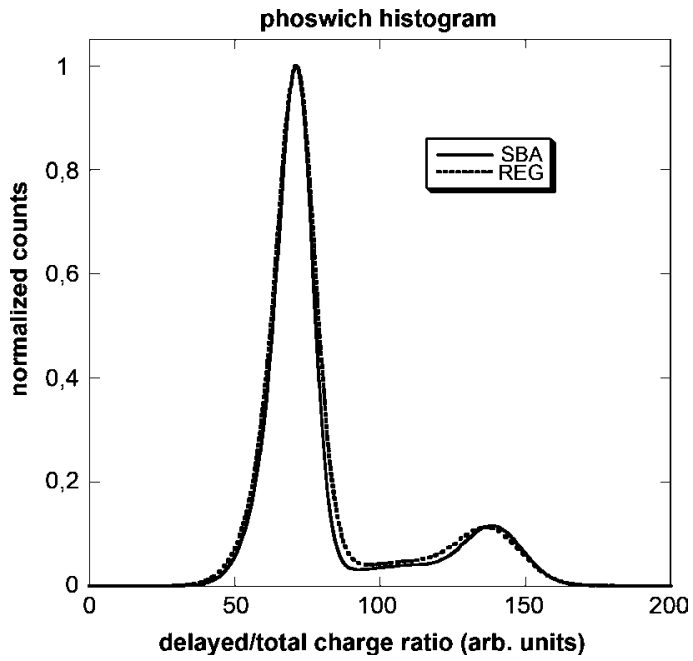

Fig. 6. Phoswich histograms for the SBA and the regular tubes: A slight improvement can be observed for the SBA tube.

number of events. Therefore, the higher peak at the left represents those events in which the tail of the pulse had very little charge (fast decay pulse: LYSO) while the lower peak at the right corresponds to those pulses in which the ratio between the full charge integral and the delayed one is much higher (slow decay pulse: GSO). Sharper peaks indicate better performance of the DOI identification procedure. The SBA phoswich diagram looks marginally improved.

\section{Discussion AND CONCLUSIONS}

The single most important factor determining energy resolution is the number of photoelectrons, $\mathrm{Ne}$, that is generated from the incident scintillation light flash by the photocathode material [1]. For a given scintillator crystal geometry, reflector material and surface treatment, (absolute) energy resolution scales with $\sqrt{\mathrm{Ne}}$ and the more commonly used relative energy resolution (expressed in\%) scales with $1 / \sqrt{\mathrm{Ne}}$. The SBA photocathodes possess an about 1.44 times higher conversion efficiency than regular BA photocathodes: $34.5 \%$ vs. $24 \%$ at $400 \mathrm{~nm}$, according to Fig. 1. We can, therefore, expect energy resolution to improve by a factor $\sqrt{1.44}=1.2$ with the new SBA PMTs. Indeed, the Fig. 5 shows that the energy resolution values of $17.3 \%$ and $16.8 \%$ ( mean $=17.05 \%$ ) for the two SBA modules are considerably better than the results for the two detectors with BA photocathodes (20.1\% and $20.6 \%$, mean $=20.35 \%$ ). This improvement in average energy resolution by a factor of 1.19 agrees well with expectation, and a boxed factorial ANOVA analysis shows that it is statistically significant $(p<0.0001)$. Similar resolution improvements have been reported for single scintillators ( $\mathrm{LaBr}_{3}$ and others) coupled to a non-position-sensitive R7600U PMTs with UBA photocathodes by [12].

The intrinsic spatial resolution also benefits from the improved photoelectron statistics provided by the SBA tubes. Numerical evidence for the higher spatial resolution is given by the average peak-to-valley ratios (Table I) in the central row profiles (Fig. 3) of the flood images. The peak-to-valley ratios obtained with the SBA PMTs (8.6 and 7.8, mean $=8.2)$ exceed those of the regular PMTs $(6.9$ and 7.0 , mean $=6.95)$ by a factor of 1.18. Since the background remains essentially the same (Fig. 3), we find an almost identical improvement factor as with the energy resolution above. As the peaks are more or less Gaussian in nature, it means that their width decreases to the same extent that the peak rises. Thus, our result confirms that intrinsic spatial resolution, like energy resolution, also scales with $1 / \sqrt{\mathrm{Ne}}$, i.e., varies according to the increasing photoelectron number [13].

An indirect indication of the higher spatial resolution of the SBA tubes can also be found in the "connect-the-dot" pattern (Fig. 2). This faint pattern is caused by inter-crystal scatter events between neighboring crystals [11], [14] and is readily visible in the flood images of the SBA detectors whereas it almost fades into the background in the slightly poorer resolution flood images of the regular detectors.

Regarding the DOI capability, the "phoswich" histograms (Fig. 6) show a marginal improvement on the DOI performance, which was already very high (97\%) as reported in [15].

The detector modules with SBA photocathodes possess an excellent packing coefficient of 0.197 (Table I), which is better by a factor of 1.38 than the (already good) value of 0.271 for the regular modules, suggesting that even smaller crystals than the $1.45 \mathrm{~mm} \times 1.45 \mathrm{~mm}$ crystals used here could be resolved. However, quantifying the crystal footprint reduction should be done experimentally or by simulations, since a smaller crystal generally has a reduced light output, a loss that can be partially compensated by the SBA higher QE. The packing coefficient combines both average spatial resolution and average spacing between the crystal centers. The lower panel in Fig. 4 shows that although the apparent crystal spacing is very similar for both tubes, the SBA shows a more uniform spacing (less dispersion on the intercrystal distances), which is an indication of a better spatial linearity. The improvement in the packing coefficient is larger than can be expected from the better spatial resolution alone. We think it may also be related to the better spatial linearity and larger field-of-view associated with the R8900-C12 PMT. The SBA photocathode material was only available for the new R8900-C12 PMT and not the older R8520-C12 PMT. Whereas these photomultipliers are equivalent for the most part, a comparison of Hamamatsu's data sheets reveals subtle differences such as a larger effective photocathode area $(23.5 \mathrm{~mm} \times 23.5 \mathrm{~mm}$ for the R8900-C12 versus $22 \mathrm{~mm} \times 22 \mathrm{~mm}$ for the R8520-C12 PMT) and an improved anode plate geometry. Whereas the R8520-C12 has $3.5 \mathrm{~mm}$ wide anode plates in the center and $2.5 \mathrm{~mm}$ wide anode plates at the edges, the R8900-C12 data sheet shows all anode plates having the same width now. The combined effect of these changes seems to be responsible for the fact that the field flood images (Fig. 2) and the central row profiles (Fig. 4) show more evenly spaced crystals and much reduced edge packing effects with the SBA R8900-C12 PMTs than with the regular R8520-C12 PMTs.

We conclude that the superior performance of the SBA tube may enable the use of arrays with a larger number of crystals of smaller footprint, thus improving the detector intrinsic spatial resolution without degrading the energy resolution or the DOI discrimination capability. Since this reduction of the crystal cross section will, in turn, reduce the amount of light 
extracted from it, the minimum crystal size that can be used without deteriorating the detector characteristics has to be further investigated. In any case, it is expected that the UBA version of these PMTs will perform even better on these terms.

\section{ACKNOWLEDGMENT}

The authors thank SEDECAL (Madrid, Spain) for providing part of the materials and tools used on this experiment.

\section{REFERENCES}

[1] Radiation Detection and Measurement, G. F. Knoll, Ed., 3rd ed. New York: Wiley, 2000, pp. 329-332.

[2] K. K. Hamamatsu-Photonics, Hamamatsu Super Bialkali Photocathodes, 2006.

[3] J. J. Vaquero, J. M. Udias, S. Espana, and M. Desco, "Effects of the super bialkali photocathode on the performance characteristics of a position-sensitive depth-of-interaction PET detector module," in Proc. IEEE Nuclear Science Symp. Conf. Rec., 2008, pp. 4945-4947.

[4] J. Seidel, J. J. Vaquero, S. Siegel, W. R. Gandler, and M. V. Green, "Depth identification accuracy of a three-layer phoswich PET detector module,” IEEE Trans. Nucl. Sci., vol. 46, no. 3, pp. 485-490, Jun. 1999.

[5] J. Seidel, J. J. Vaquero, and M. V. Green, "Resolution uniformity and sensitivity of the NIH ATLAS small animal PET scanner: Comparison to simulated LSO scanners without depth-of-Interaction capability," IEEE Trans. Nucl. Sci., vol. 50, no. 5, pp. 1347-1350, Oct. 2003.

[6] S. Siegel, R. W. Silverman, Y. Shao, and S. R. Cherry, "Simple charge division readouts for imaging scintillator arrays using a multi-channel PMT,” IEEE Trans. Nucl. Sci., vol. 43, no. 3, pp. 1634-1641, Jun. 1996.
[7] V. Popov, S. Majewski, and A. G. Weisenberger, "Readout electronics for multianode photomultiplier tubes with pad matrix anode layout," in Proc. IEEE Nuclear Science Symp. Conf. Rec., Oct. 19-25, 2003, vol. 3, pp. 2156-2159.

[8] V. Popov, S. Majewski, A. G. Weisenberger, and R. Wojcik, “Analog readout system with charge division type output," in Proc. IEEE Nuclear Science Symp. Conf. Rec., Nov. 4-10, 2001, vol. 4, pp. 1937-1940.

[9] J. J. Vaquero, J. Seidel, S. Siegel, and M. V. Green, “A depth-encoding PET detector module with improved spatial sampling," in Proc. IEEE Nuclear Science Symp. Medical Imaging Conf., 1998, pp. 1258-1258.

[10] J. Seidel, J. J. Vaquero, S. Siegel, W. R. Gandler, and M. V. Green, "Depth identification accuracy of a three layer phoswich PET detector module," IEEE Trans. Nucl. Sci., vol. 46, no. 3, pp. 485-490, Jun. 1999.

[11] J. J. Vaquero, J. Seidel, S. Siegel, W. R. Gandler, and M. V. Green, "Performance characteristics of a compact position-sensitive LSO detector module,” IEEE Trans. Med. Imag., vol. 17, pp. 967-978, Dec. 1998.

[12] N. M. Uzunov et al., "Positioning accuracy and precision of a three-degrees-of-freedom manipulator guided by tilting-collimator scintillation camera,” in Proc. IEEE Nuclear Science Symp. Conf. Rec., Dresden, Germany, 2008.

[13] M. V. Green and J. Seidel, "Single photon imaging," in Nuclear Medicine Diagnosis and Therapy, J. C. Harbert, Ed. et al. New York: Thieme Medical, 1996.

[14] P. Vaska, S. P. Stoll, C. L. Woody, D. J. Schlyer, and S. Shokouhi, "Effects of intercrystal crosstalk on multielement LSO/APD PET detectors," IEEE Trans. Nucl. Sci., vol. 50, no. 3, pp. 362-366, Jun. 2003.

[15] Y. Wang, J. Seidel, B. M. Tsui, J. J. Vaquero, and M. G. Pomper, "Performance evaluation of the GE healthcare eXplore VISTA dual-ring small-animal PET scanner,” J. Nucl. Med., vol. 47, pp. 1891-1900, Nov. 2006. 